\title{
Michel Venne
}

Directeur général, Institut du Nouveau Monde, Montréal

(2007)

\section{"Le Québec vire-t-il à droite?”}

Un document produit en version numérique par Jean-Marie Tremblay, bénévole, professeur de sociologie au Cégep de Chicoutimi

Courriel: jean-marie tremblay@uqac.ca

Site web pédagogique : http://www.uqac.ca/jmt-sociologue/

Dans le cadre de: "Les classiques des sciences sociales" Une bibliothèque numérique fondée et dirigée par Jean-Marie Tremblay, professeur de sociologie au Cégep de Chicoutimi Site web: http://classiques.uqac.ca/

Une collection développée en collaboration avec la Bibliothèque Paul-Émile-Boulet de l'Université du Québec à Chicoutimi

Site web: http://bibliotheque.uqac.ca/ 


\section{Politique d'utilisation de la bibliothèque des Classiques}

Toute reproduction et rediffusion de nos fichiers est interdite, même avec la mention de leur provenance, sans l'autorisation formelle, écrite, du fondateur des Classiques des sciences sociales, Jean-Marie Tremblay, sociologue.

Les fichiers des Classiques des sciences sociales ne peuvent sans autorisation formelle:

- être hébergés (en fichier ou page web, en totalité ou en partie) sur un serveur autre que celui des Classiques.

- servir de base de travail à un autre fichier modifié ensuite par tout autre moyen (couleur, police, mise en page, extraits, support, etc...),

Les fichiers (.html, .doc, .pdf., .rtf, .jpg, .gif) disponibles sur le site Les Classiques des sciences sociales sont la propriété des Classiques des sciences sociales, un organisme à but non lucratif composé exclusivement de bénévoles.

Ils sont disponibles pour une utilisation intellectuelle et personnelle et, en aucun cas, commerciale. Toute utilisation à des fins commerciales des fichiers sur ce site est strictement interdite et toute rediffusion est également strictement interdite.

\section{L'accès à notre travail est libre et gratuit à tous les utilisateurs. C'est notre mission.}

Jean-Marie Tremblay, sociologue

Fondateur et Président-directeur général, LES CLASSIQUES DES SCIENCES SOCIALES. 
Cette édition électronique a été réalisée par Jean-Marie Tremblay, bénévole, professeur de sociologie au Cégep de Chicoutimi à partir de :

\section{Michel Venne}

[Directeur général, Institut du Nouveau Monde, Montréal.]

“Le Québec vire-t-il à droite ?”

Introduction à l'édition 2008 de L’Annuaire du Québec., pp. 12-21 Ouvrage sous la direction de Michel Venne et Miriam Fahmy Une publication de l'INM aux Éditions Fides, 2007, Montréal, 480 pages.

[Autorisation formelle accordée par l'auteur le 14 novembre 2007 de diffuser ce texte dans Les Classiques des sciences sociales.]

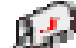

Courriel : michel.venne@inm.qc.ca

Polices de caractères utilisée :

Pour le texte: Times New Roman, 14 points.

Pour les citations : Times New Roman, 12 points.

Pour les notes de bas de page : Times New Roman, 12 points.

Édition électronique réalisée avec le traitement de textes Microsoft Word 2004 pour Macintosh.

Mise en page sur papier format : LETTRE (US letter), 8.5’’ x 11'’)

Édition numérique réalisée le 14 novembre 2007 à Chicoutimi, Ville de Saguenay, province de Québec, Canada.

\section{Fait avec}

Macintosh 


\section{Michel VENNE}

Directeur général, Institut du Nouveau Monde, Montréal

\section{“Le Québec vire-t-il à droite ?”}
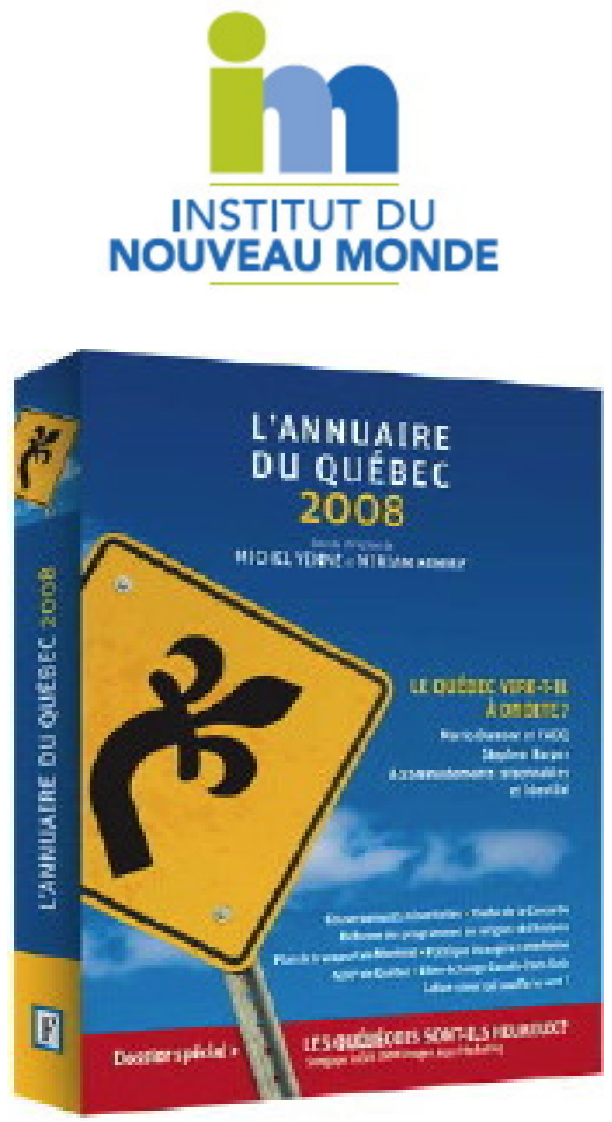

Introduction à l'édition 2008 de L’Annuaire du Québec., pp. 12-21. Sous la direction de Michel Venne et Miriam Fahmy Une publication de l'INM aux Éditions Fides, 2007, Montréal, 480 pages. 


\section{Table des matières}

Le Québec est-il de gauche?

Le résultat des élections

L’équilibre Gauche-Droite n'est pas modifié

Les positions idéologiques des parties

L’identité et la question nationale

Et pourtant, ils conservent le coeur à gauche...

Les accommodements raisonnables

Pour un État généreux

Tableau 1. Équilibre Gauche/Droite. Résultats des élections de 2003 et de 2007.

Tableau 2. Équilibre Gauche/Droite. Résultats des élections de 2003 et de 2007 (en pourcentage des suffrages exprimés) 
Michel Venne

“Le Québec vire-t-il à droite ?”

Introduction à l'édition 2008 de L’Annuaire du Québec., pp. 12-21. Sous la direction de Michel Venne et Miriam Fahmy Une publication de l'INM aux Éditions Fides, 2007, 480 pages.
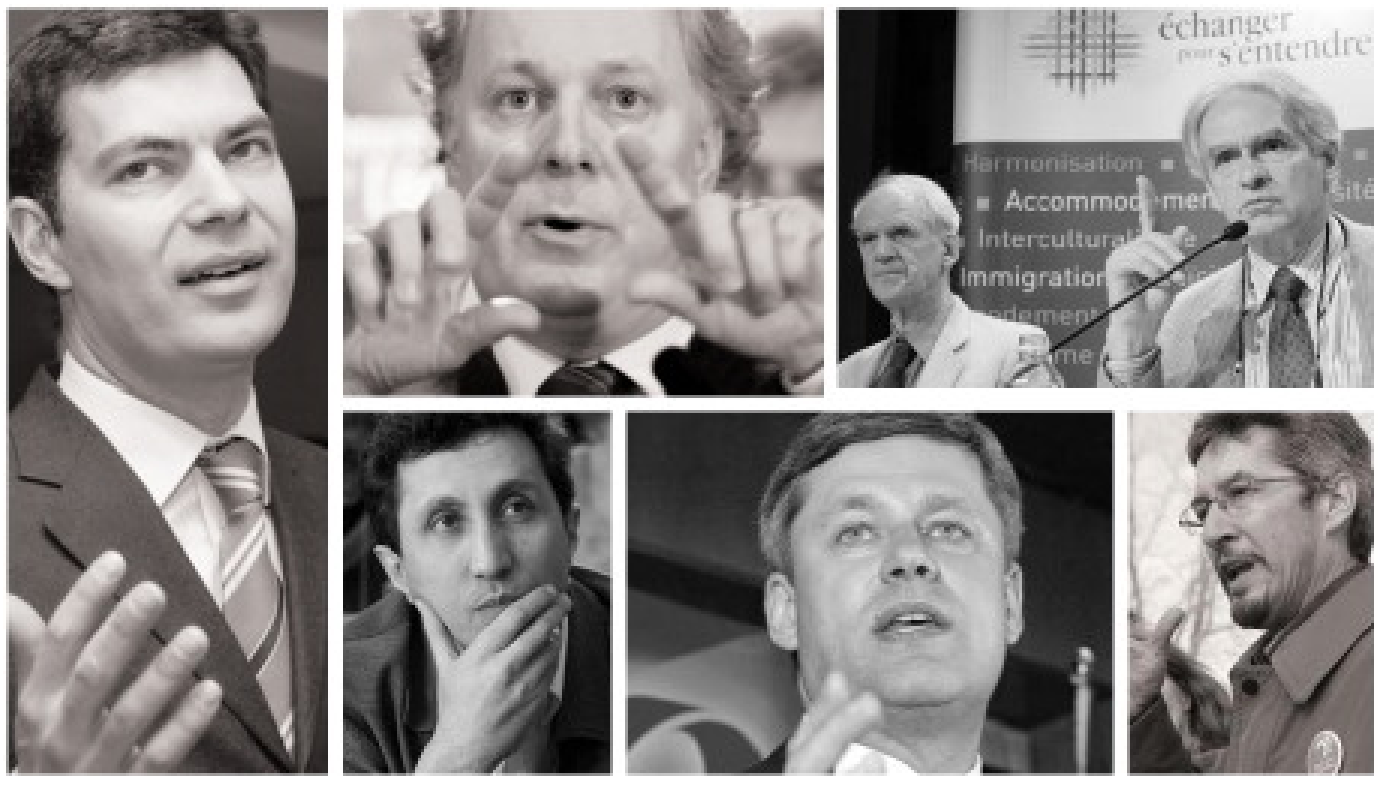

Le Québec vire-t-il à droite ? La population tourne-t-elle le dos à la social-démocratie emblématique de la Révolution tranquille des années 1960 et 1970 en accordant un appui sans précédent à l’Action démocratique du Québec aux élections du 26 mars 2007 ? Assiste-t-on à une forme de régression conservatrice et de repli sur soi, symbolisée par la vague de protestations contre les accommodements raisonnables?

Nous avons cherché à faire la part des choses. Nos conclusions sont nuancées. Si les élections du 26 mars (voir le dossier en page 23) ouvrent la possibilité d'un réalignement dans l'univers partisan, elles ne témoignent pas en soi d'une dérive conservatrice. En fait, 
l'équilibre des forces partisanes entre la gauche et la droite n'a pas bougé substantiellement entre 2003 et 2007.

Pendant qu'un demi-million d'électeurs de plus qu'en 2003 choisissaient l'ADQ de Mario Dumont et ce, largement au détriment du Parti libéral de Jean Charest, un quart de million mettaient leur confiance dans deux partis situés à gauche du PQ : Québec solidaire de Françoise David et Amir Khadir et le Parti vert de Scott Mackay.

En outre, plusieurs enquêtes d'opinion indiquent que les Québécois soutiennent de façon très majoritaire une présence forte du gouvernement dans les services aux citoyens, la distribution de la richesse et l'encadrement de l'économie. Cette opinion est encore plus marquée chez les jeunes (entre autres ceux qui ont rédigé la Grande Déclaration dans le cadre des activités de l'INM et que nous publions dans ce livre), ce qui donne une indication des tendances futures.

Les citoyens, par contre, sont déçus de l'action de leurs dirigeants politiques. Trente pour cent des électeurs ne se sont pas prévalus de leur droit de vote en 2007. La conjoncture témoigne davantage d'une déception à l'endroit de la performance de l'État providence qui s'essouffle dans sa forme historique, que d'un virage à droite.

Il est vrai toutefois que les préférences des citoyens évoluent au fur et à mesure que le niveau de vie augmente au Québec. En outre, les écarts de richesse s'accentuent. Les riches sont plus riches et possèdent plus de moyens d'exprimer leurs volontés que les petits salariés qui gagnent tout juste de quoi vivre, tandis que les syndicats, qui demeurent les organisations les plus puissantes de la gauche, sont largement discrédités dans l’opinion publique.

De son côté, la droite intellectuelle s’est donné au cours de la dernière décennie des organisations, des publications et des porte-parole omniprésents dans l'espace public, ce qui crée l'impression d'un virage à droite. 
Enfin, cette bataille idéologique se déroule dans un contexte international propice à une demande croissante de sécurité reléguant au second plan l'appétit pour les grands projets de société. Au niveau national, le débat gauche-droite a repris le dessus sur la question nationale qui, toutefois, demeure beaucoup plus structurante de l'univers politique qu'on veut bien l'admettre en certains quartiers et explique d'ailleurs en partie les succès récents de l’ADQ.

\section{Le Québec est-il de gauche?}

$\underline{\text { Retour à la table des matières }}$

Avant d'examiner si la société québécoise vire à droite, il faut voir jusqu'à quel point elle était à gauche. Le Québec est une société foncièrement libérale. Le socialisme n'y a jamais pris solidement racine, sauf dans des milieux restreints et durant une brève période. La gauche n’a jamais fait de percée significative dans le champ électoral.

Longtemps, l'Union nationale de Maurice Duplessis a dominé le Québec politique. Les conservateurs de Brian Mulroney ont triomphé au niveau fédéral pendant neuf ans de 1984 à 1993. Deux anciens ministres de ces gouvernements conservateurs, Lucien Bouchard et Jean Charest, ont ensuite gouverné le Québec pendant près de dix ans depuis ce temps.

La relative pauvreté du Québec par rapport au reste de l'Amérique du Nord et des francophones par rapport aux anglophones ici même au Québec, ont incité les Québécois à se serrer les coudes, cela est vrai. La Révolution tranquille fut largement inspirée par une volonté de rattrapage à cet égard, par la création d'un système d'éducation digne de ce nom, l'adhésion au principe d'un système public 
d'assurance-maladie, le développement d'une fonction publique professionnelle, la prise en main de notre économie.

Ce rattrapage a pu être réalisé grâce à une alliance entre les classes sociales et entre les générations qui reste tout à fait possible aujourd'hui si le leadership politique en faisait une priorité.

Le Québec copiait alors bien davantage le Royaume-Uni et la France, tout en composant avec son voisin américain, que des pays du bloc communiste. La social-démocratie québécoise n'est même jamais arrivée à la hauteur de celle pratiquée dans les pays scandinaves. Nos programmes sociaux sont encore aujourd'hui beaucoup moins généreux que ceux du Danemark ou de la Suède.

Les Québécois sont partisans du libre-échange et d'une mondialisation bien maîtrisée. Notre appui aux causes environnementales est plus que mitigé,comme vient de le démontrer mon collègue François Cardinal dans son livre Le Mythe du Québec vert (Éditions Voix parallèles).

Et puis, nous nous comportons encore, et c'est normal, comme une société minoritaire dans un continent parfois hostile à notre identité particulière, ce qui induit des attitudes défensives. Les Québécois ont peut-être le coeur à gauche, mais ils gardent le portefeuille à droite.

\section{Le résultat des élections}

Retour à la table des matières

On ne peut pas parler d'un virage conservateur au niveau fédéral. Les progrès du Parti conservateur au Québec, aux élections fédérales de janvier 2006, furent bien davantage le résultat du rejet du Parti libéral de Paul Martin, dans la foulée du scandale des commandites. Ils peuvent aussi être imputés à la volonté de nombre d'électeurs de voter 
pour des candidats qui participeraient au pouvoir, contrairement à ceux du Bloc québécois qui sont condamnés à l'opposition. En outre, la participation électorale était demeurée faible, à $64 \%$. Le Bloc québécois, toujours dirigé par l'ancien syndicaliste Gilles Duceppe, était demeuré premier en pourcentage du vote et en nombre de sièges, tandis que le NPD gagnait 100000 votes et le Parti vert, 38000.

Aux élections partielles de septembre, le Bloc a conservé un siège, le Parti conservateur en a arraché un au Bloc, grâce à une candidature locale forte, et le NPD a ravi un château fort au libéraux.

Il faut analyser les élections provinciales du 26 mars 2007 avec la même circonspection. En nombre de sièges, l'ADQ a connu une progression fulgurante, emportant 41 sièges contre 4 en 2003. Ces gains ont été réalisés principalement au détriment du Parti libéral de Jean Charest. Nous restons majoritairement dans la même famille idéologique. Au tableau des sièges, l'ADQ fait un saut de 1000\%, en multipliant par dix le nombre d'élus. Mais ce parti n'a obtenu l'appui que d'un électeur inscrit sur cinq! Ceux qui ont parlé d'un tsunami adéquiste ne savent pas compter.

À l'opposé, aucun des deux tiers partis situés à la gauche du PQ, Québec Solidaire et le Parti vert, n'est représenté à l'Assemblée nationale. Le chiffre zéro apparaît à côté de leurs noms au tableau des élus. Pourtant, 300000 personnes ont voté pour eux, soit près de $8 \%$ de l'électorat.

Avec un mode de scrutin proportionnel, l'une des nouvelles de la soirée électorale eut été l'entrée au Parlement des premiers députés verts de l'histoire du Québec, tandis que Françoise David et Amir Khadir croiseraient le fer avec les autres chefs de parti quotidiennement au Salon bleu.

Les résultats électoraux ne traduisent pas parfaitement l'humeur et les choix idéologiques des électeurs. 


\section{L’équilibre Gauche-Droite n’est pas modifié}

$\underline{\text { Retour à la table des matières }}$

La distribution des sièges à l'Assemblée nationale et le pourcentage de votes obtenu aux élections montrent la supériorité des partis situés à la droite du centre de l'échiquier politique. Pour les fins de l'analyse, je place le Parti libéral de Jean Charest et l'ADQ de Mario Dumont dans ce groupe. Le Parti québécois, Québec solidaire et le Parti vert sont mis à la gauche du centre.

Il est vrai que cette division gauche / droite des partis politiques n’est pas parfaite,les partis n'étant pas des blocs monolitiques.Mais elle permet de constater que l'équilibre qui existait en 2003 n'a pas été modifié par les élections de 2007. Il suffit d'additionner les votes obtenus par le PLQ et par l'ADQ d'un côté, et ceux récoltés par les trois autres partis de l'autre, pour voir que la progression de l'appui à droite n'est pas plus importante que la progression des appuis à gauche.

Globalement, la droite recueille les deux tiers des votes et la gauche un tiers. Mais c'était déjà le cas en 2003 (voir les tableaux 1 et 2).

Au-delà des mouvements dans la répartition des sièges, l'équilibre idéologique de la société n’a pas foncièrement changé en quatre ans.

\section{Les positions idéologiques des partis}

$\underline{\text { Retour à la table des matières }}$

Pour enrichir l'analyse, il faut examiner de plus près les raisons qui ont conduit les électeurs à se comporter comme ils l'ont fait. 
Les deux faits déterminants de la campagne sont l'effondrement électoral des libéraux et l'échec cuisant d'André Boisclair à relancer son parti après la débâcle de 2003. Le PQ avait perdu un demi-million de votes en 2003.

Les libéraux ont terminé troisièmes chez les francophones et ont perdu de nombreux votes chez les non-francophones, notamment au profit du Parti vert. L'insatisfaction de la population à l'endroit de ce gouvernement était manifeste.

Quant à André Boisclair, son inexpérience, son manque de jugement, le sketch Brokeback Mountain,sa consommation de cocaïne lorsqu'il était ministre, son incapacité à recruter l'équipe de rêve qu'il avait promise, son ambiguïté quant au programme du parti notamment à l'égard de la tenue d'un référendum, ont miné sa capacité de convaincre les électeurs perdus en 2003 de revenir au bercail en 2007. Les jeunes qui l'avaient appuyé durant la course au leadership se sont volatilisés.

TABLEAU 1 : ÉQUILIBRE GAUCHE / DROITE Résultats des élections de 2003 et de 2007

$\underline{\text { Retour à la table des matières }}$

\begin{tabular}{|l|l|l|l|l|l|l|l|}
\hline & Droite & & & & Gauche & & \\
\hline & 2003 & 2007 & Différence & & 2003 & 2007 & Différence \\
\hline PLQ & 1756000 & 1314000 & -442000 & PQ & 1269000 & 1125000 & -144000 \\
\hline ADQ & 694000 & 122400 & +530000 & $\begin{array}{l}\text { QS } \\
\text { (UFP) }\end{array}$ & 40000 & 144000 & +104000 \\
\hline & & & & PV & 17000 & 153000 & +136000 \\
\hline TOTAL & & & +88000 & & & & +96000 \\
\hline
\end{tabular}

Source : Directeur général des élections 
Mario Dumont a profité de la conjoncture. Pour y arriver, il a dû toutefois ajuster considérablement son discours.

Aux élections de 2003, la popularité de son parti atteignait les $40 \%$ dans les sondages quelques mois avant le vote. Il a finalement récolté $18 \%$ des suffrages. Le dégonflement de l'appui adéquiste fut le fait saillant de cette campagne. Les électeurs avaient rejeté l'approche conservatrice de l'ADQ pour lui préférer celle des libéraux qui avaient mené leur campagne en promettant de réinvestir dans la santé et en brandissant les valeurs sociales libérales colligées dans un livre par le défunt Claude Ryan.

\section{TABLEAU 2 : ÉQUILIBRE GAUCHE / DROITE \\ Résultats des élections de 2003 et de 2007 \\ (en pourcentage des suffrages exprimés)}

$\underline{\text { Retour à la table des matières }}$

\begin{tabular}{|l|l|l|l|l|l|}
\hline & Droite & & & Gauche & \\
\hline & 2003 & 2007 & & 2003 & 2007 \\
\hline PLQ & $46 \%$ & $33,1 \%$ & PQ & $33,2 \%$ & $28,3 \%$ \\
\hline ADQ & $18,2 \%$ & $30,8 \%$ & QS (UFP) & $1 \%$ & $3,6 \%$ \\
\hline & & & PV & $0,4 \%$ & $3,9 \%$ \\
\hline TOTAL & $64,2 \%$ & $63,9 \%$ & & $34,7 \%$ & $35,8 \%$ \\
& & & & & \\
\hline
\end{tabular}

Source : Directeur général des élections

Quatre ans plus tard, le programme adéquiste a été passablement recentré. Si le parti proposait l'abolition des commissions scolaires, il ne proposait plus la privatisation du financement des écoles par des bons d'études. Il avait abandonné l'idée du taux unique d'imposition. 
Son programme comportait même des réinvestissements publics pour la culture et pour l'éducation. Il s'engageait à ne pas augmenter les impôts, mais pas à les diminuer. L’ADQ de 2007 demeurait populiste, mais était revenue du conservatisme primaire. Il y avait moins de risques à voter pour lui.

\section{L’identité et la question nationale}

$\underline{\text { Retour à la table des matières }}$

En 2003, Mario Dumont avait donné l'impression d'abandonner le combat national des Québécois. Il avait prononcé un discours à Toronto dans lequel il se mettait à genoux devant le reste du pays, promettant de mettre fin à l'ère des sempiternelles revendications du Québec. Tous l'avaient vu, photographié devant un immense drapeau du Canada en faisant des clins d'oeil aux pontes de Bay Street.

Entre les deux élections, Mario Dumont a changé son fusil d'épaule. Son programme prônait désormais formellement la création de l'État autonome du Québec au sein de la fédération canadienne. Le statut proposé est une quasi-souveraineté, le siège à l'ONU en moins. Et encore!

Plusieurs députés adéquistes ne cachent d'ailleurs pas le maintien de leur appui à l’idée de la souveraineté du Québec.

La controverse sur les accommodements raisonnables a contribué à consolider la position de Mario Dumont comme le défenseur de la société distincte francophone non seulement contre Ottawa mais contre les intégristes islamistes. Le tout en évitant un référendum à court terme. 


\section{Et pourtant, ils conservent le coeur à gauche...}

Pierre Drouilly montre comment le vote se répartit au sein de la population et des régions du Québec dans l'article qu'il publie dans ce livre. La base électorale de l'ADQ en 2003 était celle de ce que le sociologue appelle le Québec tranquille et le Québec réfractaire, concentré autour de Québec. La Beauce en étant un bel exemple. Cette base électorale s'étend à certaines régions du centre du Québec. C'est la même base électorale qui a soutenu le Parti conservateur de Stephen Harper.

À cette base électorale s'est ajoutée en 2007 une autre frange d'électeurs habitant les banlieues nord et sud de Montréal et plus largement dans un croissant allant de l'Outaouais jusqu'à la région Chaudière-Appalaches. La zone dans laquelle l'ADQ a effectué une percée importante n'est pas celle des anciennes banlieues, plus rapprochées de Montréal, peuplées de baby-boomers, mais celle des deuxièmes et troisièmes couronnes, où ce sont installées de jeunes familles, dont les revenus sont moins élevés, obligées de s'éloigner de la ville pour trouver une maison encore abordable. Ces jeunes familles sont en train de s'installer dans la vie. Elles sont préoccupées en particulier par le sort de leurs enfants. Et elles se sentent empêchées, contraintes. Une bonne partie d'entre elles ont tout juste assez de revenus pour subvenir à leurs besoins. Leur taux d'endettement est élevé. Un accident surviendrait, une récession économique, et elles se retrouveraient à la rue. Les deux conjoints travaillent. La journée commence tôt et se termine tard. Ces gens-là sont habitués aussi à prendre leurs responsabilités. Mais elles s'attendent à ce que l'État assume aussi les siennes (voir notre sondage sur le bonheur). 
Or, ces nouveaux banlieusards s'installent dans des zones où les services de santé sont insuffisants pour suivre la croissance démographique récente, où les écoles ne répondent pas toujours aux standards attendus, où les listes d'attente pour obtenir une place en CPE sont longues, les transports publics sont inefficaces et les routes, mal entretenues. Ces gens-là ne sont pas contre l'État. Ils sont déçus de la façon dont l'État rempli ses obligations. Et ils en mettent la responsabilité sur les épaules des deux principaux partis qui se sont échangé le pouvoir depuis trente ans. Le programme de l'ADQ avait des réponses simples à leurs besoins et leur offrait une solution de rechange. Leur attitude est plus pragmatique qu'idéologique.

\section{Les accommodements raisonnables}

$\underline{\text { Retour à la table des matières }}$

La crise des accommodements raisonnables (voir le dossier en page 121) a plongé le Québec dans un grand débat existentiel. Certains ont vu dans l'expression de fortes oppositions à des accommodements accordés à des groupes religieux, un repli conservateur, et donc une forme de virage à droite.

L’Institut du Nouveau Monde a organisé, durant l'hiver 2007, un grand débat auquel ont participé 1500 citoyens et experts partout au Québec. De cet exercice est ressorti le besoin de réaffirmer nos repères identitaires. Cette volonté n'a cependant d'aucune manière entamé leur appui à la reconnaissance du pluralisme au sein de notre société et à la politique d'intégration des immigrants (et non d'assimilation) en vigueur depuis vingt ans au Québec.

Il est vrai qu'une majorité de Québécois s’opposent à la plupart des formes d'accommodements reliées aux différences religieuses : port du voile et du kirpan, espaces de prières dans les institutions, etc. Il 
faut noter que les 15-24 ans y sont par contre largement favorables. C’est qu'ils ont grandi dans les écoles pluriethniques de la loi 101.

Mais à quoi tient cette opposition? Est-elle de gauche ou de droite ? Je crois que nous voyons s'exprimer deux attitudes contradictoires. L'une défendant des valeurs progressistes et l'autre plaidant pour un retour à des idéaux d'antan.

La plupart des gens qui s’opposent à ces pratiques s'opposent en fait à ce qu'ils perçoivent comme contraire aux valeurs progressistes et libérales chères au Québec. La présence croissante de l'islam au Québec les inquiète parce qu'ils craignent de voir triompher ici les intégristes à l'oeuvre ailleurs dans le monde. Ils se portent à la défense de valeurs comme l'égalité entre les hommes et les femmes, s'opposent à la violence inhérente à certaines pratiques dont ils ont entendu parler dans certains pays musulmans comme la lapidation des femmes adultères et protègent ce qu'ils considèrent comme un acquis social: la séparation de l'Église et de l'État. Il s’agit d'une expression de valeurs de gauche.

Un autre groupe de personnes réagit aux accommodements raisonnables en proposant une forme de retour en arrière, notamment par la réhabilitation de la religion catholique. Des militants catholiques frustrés d'avoir dû assister impuissants à la déconfessionnalisation des écoles, et des nationalistes frustrés de n'avoir pas fait la souveraineté, sont devenus des alliés circonstanciels. Ceux-ci expriment une volonté commune, qu'ils agissent par conviction ou par stratégie. Ils portent le risque d'une réelle régression qui ne correspondrait peut-être pas aux voeux de la majorité. 


\section{Pour un État généreux}

Les Québécois ne tournent pas massivement le dos à la socialdémocratie. Le président de la firme de sondage CROP, Alain Giguère, a eu la surprise de sa vie en prenant connaissance des résultats combinés de plusieurs enquêtes menées par son entreprise au cours des derniers mois de l'année 2006: les Québécois manifestent un très grand attachement au rôle de l'État dans les services publics, la redistribution de la richesse et l'encadrement de l'économie.

Cinquante-sept pour cent disent que le Québec doit être «le plus généreux possible dans ses programmes sociaux» contre $36 \%$ qui trouvent qu'il l'est déjà trop. Ils s'opposent à une hausse des droits de scolarité à l'université, à une hausse des tarifs d'électricité et, dans une moindre mesure, à l'imposition d'un ticket modérateur dans le domaine de la santé.

D’aucuns ont interprété ces réponses comme la manifestation de l'égoïsme de citoyens choyés ou de leur inconscience des problèmes de finances publiques. Mon interprétation est différente. Notre sondage sur le bonheur (voir le dossier en page 97) révèle que 55\% des Québécois estiment que leurs revenus sont tout juste suffisants pour répondre à leurs besoins fondamentaux. Toute contrainte financière, toute hausse de tarif supplémentaire serait intolérable.

Par contre, ils savent que les services publics ne sont pas gratuits. Pendant la campagne électorale, Jean Charest avait promis de baisser les impôts de 950 millions de dollars. Après sa réélection, formant un gouvernement minoritaire, il a réitéré cet engagement et voulut le mettre en oeuvre par le dépôt d'un nouveau budget. Un sondage a alors indiqué que si on leur donnait le choix entre baisser les impôts 
ou investir cet argent dans l'amélioration des services de santé et d'éducation, seulement $27 \%$ choisissaient la première option et $70 \%$ la seconde.

Les récents événements politiques ne peuvent nous amener à conclure à un virage à droite des Québécois. La société québécoise est déjà une société libérale. L'État providence l'a bien servi jusqu’à présent. Les Québécois ont fait des choix partisans dictés avant tout par leur déception par rapport aux dirigeants politiques et par rapport à l'inefficacité des services publics. Ils se sentent contraints et empêchés d'améliorer leur sort. Ils constatent la montée des inégalités. La stagnation du mouvement souverainiste a pour effet de produire une détente sur le front de la question nationale qui laisse le terrain aux autres enjeux. Cependant, les Québécois demeurent majoritairement nationalistes et voudront des leaders capables de défendre leur identité. À cet égard, dans le contexte de la mondialisation, bien des Québécois sont inquiets à ce sujet et ils craignent la montée de mouvements radicaux. L’époque crée un très grand appétit pour une plus grande sécurité, tant sur le plan financier, sur le plan affectif que sur le plan culturel.

Les Québécois n’appellent pas au conservatisme, ils demandent la sécurité, l’efficacité et la justice.

\section{NOTE}

1. Lire à ce sujet Drouilly, Pierre; Les élections du 23 janvier 2006, dans Venne Michel et Miriam Fahmy (dir.), L’Annuaire du Québec 2007, p. 360-370. Une version étendue de cette analyse est disponible sur le site Internet de l'Institut du Nouveau Monde, onglet Annuaire du Québec 2007.

\section{Fin du texte}

